K. Sakai

Nagoya Math. J.

Vol. 111 (1988), 111-114

\title{
QUASI-ANOSOV DIFFEOMORPHISMS AND PSEUDO-ORBIT TRACING PROPERTY
}

\author{
KAZUHIRO SAKAI
}

Let $M$ be a compact boundaryless $C^{\infty}$-manifold, and let $\operatorname{Diff}(M)$ be the space of $C^{1}$-diffeomorphisms of $M$ endowed with the $C^{1}$-topology. An Axiom $A$ diffeomorphism is said to satisfy the strong transversality condition if for every $x \in M, T_{x} M=T_{x} W^{s}(x)+T_{x} W^{u}(x)$. For an Axiom $A$ diffeomorphism, the strong transversality is a sufficient condition to be structurally stable (i.e. there is a neighbourhood $\mathscr{U} \subset \operatorname{Diff}(M)$ of $f$ such that for every $g \in \mathscr{U}$, there is a homeomorphism $h$ on $M$ satisfying $f \circ h=$ $h \circ g)$. We say that $f \in \operatorname{Diff}(M)$ is topologically stable if for every $\varepsilon>0$, there is a neighbourhood $\mathscr{U}_{\varepsilon}$ of $f$ in the set of homeomorphisms of $M$ with the $C^{0}$-topology such that for every $g \in \mathscr{U}_{\varepsilon}$, there is a continuous surjection $h$ on $M$ satisfying $f \circ h=h \circ g$ and $d(h(x), x)<\varepsilon$ for $x \in M$ (here $d$ denotes a metric compatible with the topology of $M$ ).

Let $g: X \rightarrow X$ be a homeomorphism of a compact metric space $(X, d)$. A sequence of points $\left\{x_{i}\right\}_{i=a}^{b}(-\infty \leqq a<b \leqq \infty)$ in $X$ is called a $\delta$-pseudoorbit of $g$ if $d\left(g\left(x_{i}\right), x_{i+1}\right)<\delta$ for $a \leqq i \leqq b-1$. A sequence $\left\{x_{i}\right\}$ is called to be $\varepsilon$-traced by $x \in X$ if $d\left(g^{i}(x), x_{i}\right)<\varepsilon$ holds for $a \leqq i \leqq b$. We say that $g$ has pseudo-orbit tracing property (abbrev. POTP) if for every $\varepsilon>0$ there is $\delta>0$ such that every $\delta$-pseudo-orbit of $g$ can be $\varepsilon$-traced by some point in $X$. We say that $g$ is expansive if there exists $c>0$ such that $d\left(g^{n}(x), g^{n}(y)\right) \leqq c$ for every $n \in Z$ implies $x=y$. Such a number $c$ is called an expansive constant for $g$. For the materials of topological dynamics on compact manifolds, see Morimoto [4].

It is well known that every homeomorphism on $M$ with expansivity and POTP is topologically stable, and that every topologically stable homeomorphism on $M$ of dimension $\geqq 2$ has POTP (see [4]). Every Axiom $A$ diffeomorphism $f$ satisfying the strong transversality condition is topologically stable (thus every Anosov diffeomorphism is topologically

Received November 15, 1986. 
stable) and so $f$ has POTP.

We say that $f \in \operatorname{Diff}(M)$ is quasi-Anosov if for every $0 \neq v \in T M$, the set $\left\{\left\|(T f)^{n}(v)\right\|: n \in Z\right\}$ is unbounded. A quasi-Anosov diffeomorphism is equivalent to an Axiom $A$ diffeomorphism satisfying $T_{x} W^{s}(x) \cap T_{x} W^{u}(x)$ $=\left\{0_{x}\right\}$ for every $x \in M([3])$. Obviously every Anosov diffeomorphism is quasi-Anosov and its converse is true if $\operatorname{dim} M=2$ ([3]). But it is known ([1]) that the converse is not true on a 3-dimensional manifold. Mañé proved the following

TheOREm ([3]). For $f \in \operatorname{Diff}(M)$ the following conditions are mutually equivalent;

(i) $f$ is Anosov,

(ii) $f$ is quasi-Anosov and satisfies the strong transversality condition,

(iii) $f$ is quasi-Anosov and structurally stable.

The aim of this note is to prove the following theorem related to the above results.

Theorem. Every quasi-Anosov diffeomorphism with POTP must be an Anosov diffeomorphism.

First of all we prepare a lemma that we need.

Lemma. Let $M$ be as before and let $f \in \operatorname{Diff}(M)$ be quasi-Anosov. Then there are an integer $m>0$ and a neighbourhood $\mathscr{V} \subset \operatorname{Diff}(M)$ of $f$ such that for every $g \in \mathscr{V}$ and every $0 \neq v \in T M,\left\|(T g)^{n}(v)\right\| \geqq 2\|v\|$ for some $n$ with $|n|=m$.

Proof. Since $f$ is quasi-Anosov, it is easy to see that there is $N>0$ such that for every $0 \neq v \in T M,\left\|(T f)^{n}(v)\right\| \geqq 3\|v\|$ for some $n$ with $|n|<N$. Thus following the proof of Lemma 2.3 of [2] we see that there is $m>\theta$ such that for every $0 \neq v \in T M,\left\|(T f)^{n}(v)\right\| \geqq 3\|v\|$ for some $n$ with $|n|=m$. Thus if we choose a neighbourhood $\mathscr{V} \subset \operatorname{Diff}(M)$ of $f$ such that for every $g \in \mathscr{V},\left\|(T g)^{n}-(T f)^{n}\right\|<1$ for $|n|=m$, then the conclusion of this lemma is obtained.

\section{Proof of Theorem}

Let $f: M \rightarrow M$ be a quasi-Anosov diffeomorphism with POTP. If we establish that there is a neighbourhood $\mathscr{U} \subset \operatorname{Diff}(M)$ of $f$ such that every $g \in \mathscr{U}$ has a common expansive constant, then the conclusion of our theorem is easily obtained as follows. 
Since $f$ is expansive and has POTP, $f$ is topologically stable. Thus if we choose a small $\left(C^{1}-\right)$ neighbourhood $\mathscr{U}^{\prime} \subset \mathscr{U}$ of $f$, then for every $g \in \mathscr{U}^{\prime}$, there is a continuous surjection $h: M \rightarrow M$ with $f \circ h=h \circ g$ and $d(h(x), x)<c / 3$ for all $x \in M$. Since an expansive constant for $g$ is the same as that of $f$, we see that $h$ is injective. This implies that $f$ is structurally stable, and so $f$ is Anosov.

We denote by exp the exponential map from $T M$ to $M$ determined by a Riemannian metric $\|\cdot\|$ on $T M$. Let $m>0$ and $\mathscr{V}$ be as in the lemma and put $K=\sup \left\{\left\|(T g)_{x}\right\|: x \in M, g \in \mathscr{V}\right\}$. Take and fix $\varepsilon>0$ such that $\varepsilon\left(1+K+\cdots+K^{m-1}\right)<1 / 2$. Then there are $c=c(\varepsilon, f)>0$ and a neighbourhood $\mathscr{U}(\subset \mathscr{V})$ of $f$ such that for every $g \in \mathscr{U}$,

$$
\left\|\exp _{g^{\sigma}(x)}^{-1} \circ g^{\sigma} \circ \exp _{x} v-(T g)_{x}^{o}(v)\right\| \leqq\|v\| \varepsilon \quad(x \in M)
$$

if $\|v\| \leqq c(\sigma= \pm 1)$. To get the conclusion, it is enough to see that $c$ is a common expansive constant for all $g \in \mathscr{U}$. If this is false, then there exist $x, y \in M(x \neq y)$ and $g \in \mathscr{U}$ such that $d\left(g^{n}(x), g^{n}(y)\right) \leqq c$ for $n \in Z$ (here $d$ is the metric induced by the Riemannian metric). Let $c_{1}=\sup \left\{d\left(g^{n}(x)\right.\right.$, $\left.\left.g^{n}(y)\right): n \in Z\right\}$ and take $\delta$ with $0<\delta \leqq c_{1} / 4$. Obviously $c_{1}-\delta<d\left(g^{k}(x)\right.$, $\left.g^{k}(y)\right) \leqq c_{1}$ for some $k \in Z$. Let $z=g^{k}(x), w=g^{k}(y)$ and $v=\exp _{z}^{-1} w$. Then $c_{1}-\delta<\|v\|=d(z, w)$ and $\left\|(T g)^{n}(v)\right\| \geqq 2\|v\|$ for some $n$ with $|n|=m$. We deal with only the case $\left\|(T g)^{m}(v)\right\| \geqq 2\|v\|$ (since the case $\left\|(T g)^{-m}(v)\right\| \geqq 2\|v\|$ follows in a similar way). Since $\|v\|=d(z, w) \leqq c$ we have

$$
\left\|\exp _{g(z)}^{-1} \circ g \circ \exp _{z} v-(T g)_{z}(v)\right\| \leqq\|v\| \varepsilon,
$$

and so $\left\|(T g)_{z}(v)\right\| \leqq c_{1}(1+\varepsilon)\left(\right.$ since $\left.\left\|\exp _{g(z)}^{-1} \circ g \circ \exp _{z} v\right\|=d(g(z), g(w)) \leqq c_{1}\right)$. Moreover

$$
\begin{aligned}
& \left\|\exp _{g^{2}(z)}^{-1} \circ g^{2} \circ \exp _{z} v-(T g)_{z}^{2}(v)\right\| \\
& \leqq \\
& \quad\left\|\exp _{g^{2}(z)}^{-1} \circ g^{2} \circ \exp _{z} v-(T g)_{g(z)}\left(\exp _{g(z)}^{-1} \circ g \circ \exp _{z} v\right)\right\| \\
& \quad+\left\|(T g)_{g(z)}\left(\exp _{g(z)}^{-1} \circ g \circ \exp _{z} v\right)-(T g)_{z}^{2}(v)\right\| \\
& \leqq \\
& \quad c_{1} \varepsilon+K c_{1} \varepsilon=c_{1} \varepsilon(1+K)
\end{aligned}
$$

and hence

$$
\left\|\exp _{g^{2}(z)}^{-1} \circ g^{2} \circ \exp _{z} v\right\|=d\left(g^{2}(z), g^{2}(w)\right) \leqq c_{1}
$$

implies

$$
\left\|(T g)_{z}^{2}(v)\right\| \leqq c_{1}\{1+\varepsilon(1+K)\} .
$$

By induction we have 


$$
2\|v\| \leqq\left\|(T g)_{z}^{m}(v)\right\| \leqq c_{1}\left\{1+\varepsilon\left(1+K+\cdots+K^{m-1}\right)\right\} .
$$

Thus $c_{1}-\delta<\|v\| \leqq 3 c_{1} / 4$ and we have $c_{1} / 4<\delta$. This is a contradiction.

\section{REFERENCES}

[1] J. Franks and C. Robinson, A quasi-Anosov diffeomorphism that is not Anosov, Trans. Amer. Math. Soc., 223 (1976), 267-278.

[2] J. Lewowicz, Lyapunov functions and topological stability, J. Differential Equations, 38 (1980), 192-209.

[ 3 ] R. Mañé, Quasi-Anosov diffeomorphisms and hyperbolic manifolds, Trans. Amer. Math. Soc., 229 (1977), 351-370.

[4] A. Morimoto, The method of pseudo-orbit tracing property and stability, Tokyo Univ. Seminary Notes 39, 1979. (In Japanese.)

Department of Mathematics

Tokyo Metropolitan University

Tokyo, Japan

Current address:

Kisarazu National College of Technology

2-11-1, Kiyomidai-higashi

Kisarazu-shi

Chiba-ken 292, Japan 